Slavica

bruxellensia

\section{Slavica bruxellensia}

Revue polyphonique de littérature, culture et histoire

slaves

$8 \mid 2012$

Migration(s) et Exil(s)

\title{
Jan Woleński, L'École de Lvov-Varsovie. Philosophie et logique en Pologne (1895-1939)
}

\section{Sébastien Richard}

\section{OpenEdition}

\section{Journals}

Édition électronique

URL : http://journals.openedition.org/slavica/1049

DOI : 10.4000/slavica.1049

ISSN : 2034-6395

\section{Éditeur}

Université libre de Bruxelles - ULB

\section{Référence électronique}

Sébastien Richard, « Jan Woleński, L'École de Lvov-Varsovie. Philosophie et logique en Pologne (1895-1939) », Slavica bruxellensia [En ligne], 8| 2012, mis en ligne le 25 juin 2012, consulté le 22 septembre 2020. URL : http://journals.openedition.org/slavica/1049; DOl : https://doi.org/10.4000/ slavica. 1049

Ce document a été généré automatiquement le 22 septembre 2020.

\section{cc) $(1) \odot$}

Les contenus de Slavica bruxellensia sont mis à disposition selon les termes de la Licence Creative Commons Attribution - Pas d'Utilisation Commerciale - Pas de Modification 3.0 France. 


\section{Jan Woleński, L'École de Lvov- Varsovie. Philosophie et logique en Pologne (1895-1939)}

Sébastien Richard

\section{RÉFÉRENCE}

Woleński J., L'École de Lvov-Varsovie. Philosophie et logique en Pologne (1895-1939), Vrin, coll. « Analyse et philosophie », Paris, 2011, 283 p. Traduit du polonais par Anna C. Zielińska 
1 Le présent ouvrage est la traduction de Filozoficzna szkoła lwowsko-warszawska, paru en en polonais en 1985 et déjà traduit en anglais (1989) et en russe (2004). Il s'agit assurément d'un livre désormais classique sur la philosophie de l'École de LvovVarsovie. Celle-ci y est principalement étudiée durant la période qui s'étend de 1895 à 1939, c'est-à-dire durant la période qui sépare la nomination de Kazimierz Twardowski à la chaire de philosophie de l'Université de Lvov, considérée comme l'acte de naissance de l'École de LvovVarsovie, et l'invasion de la Pologne en septembre 1939 suite au pacte Ribbentrop-Molotov, événement qui devait entraîner la mort ou la fuite de la plupart des membres de l'école polonaise de philosophie.

2 L'un des intérêts de l'ouvrage de Jan Woleński est son approche

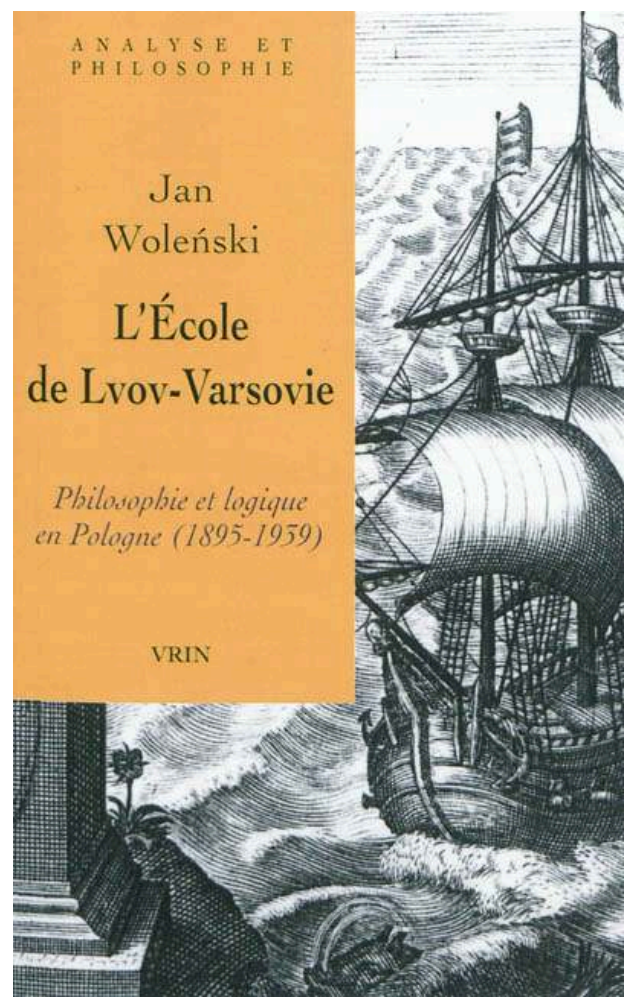
multidisciplinaire. En effet, il ne se contente pas d'exposer les doctrines philosophiques les plus importantes de certains des membres de l'École de Lvov-Varsovie, mais étudie aussi celle-ci du point de vue de l'histoire institutionnelle et de la sociologie de la connaissance. Par là, il nous offre une interprétation subtile de ce qu'a pu être l'esprit philosophique partagé par les philosophes de cette tradition, plutôt qu'une introuvable unité doctrinale.

De nos jours, l'École de Lvov-Varsovie est surtout connue pour ses importantes contributions à la logique ; que l'on songe ici aux travaux de Jan Łukasiewicz, Stanisław Leśniewski, ou encore Alfred Tarski. Or c'est l'un des intérêts du livre de Woleński que de nuancer fortement cette image de l'âge d'or de la philosophie polonaise, tout en ne sous-estimant pas la valeur qu'a eue la logique (deux chapitres lui sont consacrés) en Pologne durant cette période. L'auteur consacre ainsi de longs développements aux contributions d'auteurs, entre autres, tels que Twardowski, Kazimierz Ajdukiewicz et Tadeusz Kotarbiński (ces deux derniers étant les figures dominantes de l'École de LvovVarsovie après la Seconde Guerre mondiale) à la philosophie des sciences, l'ontologie, la psychologie, la théorie du jugement, la philosophie du langage, etc. Cette diversité des domaines philosophiques étudiés aurait certainement pu être étendue à l'esthétique (par exemple aux travaux de Władysław Tatarkiewicz), à l'éthique (un chapitre de l'ouvrage original lui était pourtant consacré), ou encore à la philosophie de la religion (par exemple les travaux de Jan Salamucha et de Józef Maria Bocheński).

Il faut souligner que la présente traduction ne reprend pas l'intégralité du volume paru originellement en polonais. Plusieurs chapitres sont ainsi omis, remaniés ou, dans le cas de ceux consacrés à la logique, remplacés par de nouveaux, issus de publications plus récentes de Woleński. À notre avis, ce choix est malheureux, car certaines des théories présentées ailleurs dans la traduction, par exemple la doctrine réiste de Kotarbiński, requièrent - pour être bien comprises - des développements logiques qui 
figuraient dans l'ouvrage original. Outre le fait que ces omissions nuisent à la compréhension de l'ensemble du livre, elles pourraient conduire à une conception erronée de l'importance des travaux philosophiques de l'École de Lvov-Varsovie. En effet, si dans le livre orignal polonais la mise en évidence des apports de la tradition philosophique polonaise à d'autres domaines que la logique était des plus salutaires, en ce qu'elle contribuait à en donner une image moins caricaturale, la logique se trouve, dans la présente traduction, presque sous-déterminée, de sorte que le lecteur pourrait avoir l'impression qu'elle n'est finalement que de peu d'importance. C'est là selon nous la conséquence la plus dommageable des choix éditoriaux de cette traduction. Il reste toutefois que, malgré ses imperfections, celle-ci rend enfin disponible en français un ouvrage de tout premier ordre sur l'École de Lvov-Varsovie.

\section{INDEX}

Index géographique : Pologne, Ukraine

Keywords : Lvov-Warsaw School, Polish philosophy

Mots-clés : école de Lvov-Varsovie, philosophie polonaise

Index chronologique : entre-deux-guerres, fin de siècle, Première Guerre mondiale, XXe siècle

Schlüsselwörter : Lemberg-Warschau-Schule, polnische Philosophie

\section{AUTEURS}

\section{SÉBASTIEN RICHARD}

Chercheur post-doctorant F.R.F.C. à l'Université Libre de Bruxelles (Belgique). Membre du Centre de recherches en philosophie. sebastien.richard@ulb.ac.be 